\title{
Egoroff's Theorem
}

\author{
Noboru Endou \\ Yasunari Shidama \\ Gifu National College of Technology \\ Japan \\ Shinshu University \\ Nagano, Japan \\ Keiko Narita \\ Hirosaki-city \\ Aomori, Japan
}

\begin{abstract}
Summary. The goal of this article is to prove Egoroff's Theorem [13]. However, there are not enough theorems related to sequence of measurable functions in Mizar Mathematical Library. So we proved many theorems about them. At the end of this article, we showed Egoroff's theorem.
\end{abstract}

MML identifier: MESFUNC8, version: $\underline{7.8 .10 \quad 4.100 .1011}$

The articles [18], [3], [15], [16], [5], [12], [22], [6], [19], [20], [8], [14], [7], [4], [17], $[1],[10],[11],[9],[21]$, and [2] provide the notation and terminology for this paper.

\section{Selected Properties of Functional Sequences}

In this paper $n, k$ are natural numbers, $X$ is a non empty set, and $S$ is a $\sigma$-field of subsets of $X$.

Next we state several propositions:

(1) Let $M$ be a $\sigma$-measure on $S, F$ be a function from $\mathbb{N}$ into $S$, and given $n$. Then $\left\{x \in X: \bigwedge_{k}(n \leq k \Rightarrow x \in F(k))\right\}$ is an element of $S$.

(2) Let $F$ be a sequence of subsets of $X$ and $n$ be an element of $\mathbb{N}$. Then (the superior set sequence of $F)(n)=\bigcup \operatorname{rng}(F \uparrow n)$ and (the inferior set sequence of $F)(n)=\bigcap \operatorname{rng}(F \uparrow n)$.

(3) Let $M$ be a $\sigma$-measure on $S$ and $F$ be a sequence of subsets of $S$. Then there exists a function $G$ from $\mathbb{N}$ into $S$ such that $G=$ the inferior set sequence of $F$ and $M(\liminf F)=\sup \operatorname{rng}(M \cdot G)$. 
(4) Let $M$ be a $\sigma$-measure on $S$ and $F$ be a sequence of subsets of $S$. Suppose $M(\bigcup F)<+\infty$. Then there exists a function $G$ from $\mathbb{N}$ into $S$ such that $G=$ the superior set sequence of $F$ and $M(\lim \sup F)=\inf \operatorname{rng}(M \cdot G)$.

(5) Let $M$ be a $\sigma$-measure on $S$ and $F$ be a sequence of subsets of $S$. Suppose $F$ is convergent. Then there exists a function $G$ from $\mathbb{N}$ into $S$ such that $G=$ the inferior set sequence of $F$ and $M(\lim F)=\sup \operatorname{rng}(M \cdot G)$.

(6) Let $M$ be a $\sigma$-measure on $S$ and $F$ be a sequence of subsets of $S$. Suppose $F$ is convergent and $M(\bigcup F)<+\infty$. Then there exists a function $G$ from $\mathbb{N}$ into $S$ such that $G=$ the superior set sequence of $F$ and $M(\lim F)=$ $\inf \operatorname{rng}(M \cdot G)$.

Let $X, Y$ be sets and let $F$ be a sequence of partial functions from $X$ into $Y$. We say that $F$ has the same dom if and only if:

(Def. 1) $\operatorname{rng} F$ has common domain.

Let $X, Y$ be sets and let $F$ be a sequence of partial functions from $X$ into $Y$. Let us observe that $F$ has the same dom if and only if:

(Def. 2) For all natural numbers $n, m$ holds $\operatorname{dom} F(n)=\operatorname{dom} F(m)$.

Let $X, Y$ be sets. One can verify that there exists a sequence of partial functions from $X$ into $Y$ which has the same dom.

Let $X$ be a non empty set and let $f$ be a sequence of partial functions from $X$ into $\overline{\mathbb{R}}$. The functor inf $f$ yielding a partial function from $X$ to $\overline{\mathbb{R}}$ is defined as follows:

(Def. 3) $\operatorname{dominf} f=\operatorname{dom} f(0)$ and for every element $x$ of $X$ such that $x \in$ dominf $f$ holds $(\inf f)(x)=\inf (f \# x)$.

Let $X$ be a non empty set and let $f$ be a sequence of partial functions from $X$ into $\overline{\mathbb{R}}$. The functor sup $f$ yields a partial function from $X$ to $\overline{\mathbb{R}}$ and is defined by:

(Def. 4) $\operatorname{domsup} f=\operatorname{dom} f(0)$ and for every element $x$ of $X$ such that $x \in$ $\operatorname{dom} \sup f$ holds $(\sup f)(x)=\sup (f \# x)$.

Let $X$ be a non empty set and let $f$ be a sequence of partial functions from $X$ into $\overline{\mathbb{R}}$. The inferior real sequence of $f$ yields a sequence of partial functions from $X$ into $\overline{\mathbb{R}}$ with the same dom and is defined by the condition (Def. 5).

(Def. 5) Let $n$ be a natural number. Then

(i) $\operatorname{dom}($ the inferior real sequence of $f)(n)=\operatorname{dom} f(0)$, and

(ii) for every element $x$ of $X$ such that $x \in \operatorname{dom}$ (the inferior real sequence of $f)(n)$ holds (the inferior real sequence of $f)(n)(x)=($ the inferior real sequence of $f \# x)(n)$.

Let $X$ be a non empty set and let $f$ be a sequence of partial functions from $X$ into $\overline{\mathbb{R}}$. The superior real sequence of $f$ yields a sequence of partial functions from $X$ into $\overline{\mathbb{R}}$ with the same dom and is defined by the condition (Def. 6).

(Def. 6) Let $n$ be a natural number. Then 
(i) $\operatorname{dom}($ the superior real sequence of $f)(n)=\operatorname{dom} f(0)$, and

(ii) for every element $x$ of $X$ such that $x \in \operatorname{dom}$ (the superior real sequence of $f)(n)$ holds (the superior real sequence of $f)(n)(x)=($ the superior real sequence of $f \# x)(n)$.

One can prove the following proposition

(7) Let $f$ be a sequence of partial functions from $X$ into $\overline{\mathbb{R}}$ and $x$ be an element of $X$. Suppose $x \in \operatorname{dom} f(0)$. Then (the inferior real sequence of $f) \# x=$ the inferior real sequence of $f \# x$.

Let $X, Y$ be sets. We see that the sequence of partial functions from $X$ into $Y$ is a function from $\mathbb{N}$ into $X \dot{\rightarrow} Y$.

Let $X, Y$ be sets, let $f$ be a sequence of partial functions from $X$ into $Y$ with the same dom, and let $n$ be an element of $\mathbb{N}$. Observe that $f \uparrow n$ has the same dom.

Next we state three propositions:

(8) Let $f$ be a sequence of partial functions from $X$ into $\overline{\mathbb{R}}$ with the same dom and $n$ be an element of $\mathbb{N}$. Then (the inferior real sequence of $f)(n)=$ $\inf (f \uparrow n)$.

(9) Let $f$ be a sequence of partial functions from $X$ into $\overline{\mathbb{R}}$ with the same dom and $n$ be an element of $\mathbb{N}$. Then (the superior real sequence of $f)(n)=$ $\sup (f \uparrow n)$.

(10) Let $f$ be a sequence of partial functions from $X$ into $\overline{\mathbb{R}}$ and $x$ be an element of $X$. Suppose $x \in \operatorname{dom} f(0)$. Then (the superior real sequence of $f) \# x=$ the superior real sequence of $f \# x$.

Let $X$ be a non empty set and let $f$ be a sequence of partial functions from $X$ into $\overline{\mathbb{R}}$. The functor liminf $f$ yielding a partial function from $X$ to $\overline{\mathbb{R}}$ is defined as follows:

$(\text { Def. } 8)^{1} \operatorname{dom} \liminf f=\operatorname{dom} f(0)$ and for every element $x$ of $X$ such that $x \in$ $\operatorname{dom} \liminf f$ holds $(\liminf f)(x)=\liminf (f \# x)$.

Let $X$ be a non empty set and let $f$ be a sequence of partial functions from $X$ into $\overline{\mathbb{R}}$. The functor $\lim \sup f$ yielding a partial function from $X$ to $\overline{\mathbb{R}}$ is defined as follows:

(Def. 9) $\operatorname{dom} \limsup f=\operatorname{dom} f(0)$ and for every element $x$ of $X$ such that $x \in$ $\operatorname{dom} \lim \sup f$ holds $(\limsup f)(x)=\lim \sup (f \# x)$.

We now state three propositions:

(11) Let $f$ be a sequence of partial functions from $X$ into $\overline{\mathbb{R}}$. Then

(i) for every element $x$ of $X$ such that $x \in \operatorname{dom} \liminf f$ holds $(\liminf f)(x)=\sup ($ the inferior real sequence of $f \# x)$ and $(\liminf f)(x)=\sup (($ the inferior real sequence of $f) \# x)$ and $(\liminf f)(x)=(\sup ($ the inferior real sequence of $f))(x)$, and

\footnotetext{
${ }^{1}$ The definition (Def. 7) has been removed.
} 
(ii) $\liminf f=\sup ($ the inferior real sequence of $f$ ).

(12) Let $f$ be a sequence of partial functions from $X$ into $\overline{\mathbb{R}}$. Then

(i) for every element $x$ of $X$ such that $x \in \operatorname{dom} \limsup f$ holds $(\limsup f)(x)=\inf ($ the superior real sequence of $f \# x)$ and $(\limsup f)(x)=\inf (($ the superior real sequence of $f) \# x)$ and $(\limsup f)(x)=(\inf ($ the superior real sequence of $f))(x)$, and

(ii) $\lim \sup f=\inf ($ the superior real sequence of $f$ ).

(13) Let $f$ be a sequence of partial functions from $X$ into $\overline{\mathbb{R}}$ and $x$ be an element of $X$. If $x \in \operatorname{dom} f(0)$, then $f \# x$ is convergent iff $(\limsup f)(x)=$ $(\liminf f)(x)$.

Let $X$ be a non empty set and let $f$ be a sequence of partial functions from $X$ into $\overline{\mathbb{R}}$. The functor $\lim f$ yielding a partial function from $X$ to $\overline{\mathbb{R}}$ is defined by:

(Def. 10) $\operatorname{dom} \lim f=\operatorname{dom} f(0)$ and for every element $x$ of $X$ such that $x \in$ $\operatorname{dom} \lim f$ holds $(\lim f)(x)=\lim (f \# x)$.

One can prove the following propositions:

(14) Let $f$ be a sequence of partial functions from $X$ into $\overline{\mathbb{R}}$ and $x$ be an element of $X$. If $x \in \operatorname{dom} \lim f$ and $f \# x$ is convergent, then $(\lim f)(x)=$ $(\limsup f)(x)$ and $(\lim f)(x)=(\liminf f)(x)$.

(15) Let $f$ be a sequence of partial functions from $X$ into $\overline{\mathbb{R}}$ with the same dom, $F$ be a sequence of subsets of $S$, and $r$ be a real number. Suppose that for every natural number $n$ holds $F(n)=\operatorname{dom} f(0) \cap \mathrm{GT}$-dom $(f(n), \overline{\mathbb{R}}(r))$. Then $\bigcup \operatorname{rng} F=\operatorname{dom} f(0) \cap \mathrm{GT}$-dom $(\sup f, \overline{\mathbb{R}}(r))$.

(16) Let $f$ be a sequence of partial functions from $X$ into $\overline{\mathbb{R}}$ with the same dom, $F$ be a sequence of subsets of $S$, and $r$ be a real number. Suppose that for every natural number $n$ holds $F(n)=\operatorname{dom} f(0) \cap$ GTE-dom $(f(n), \overline{\mathbb{R}}(r))$. Then $\bigcap \operatorname{rng} F=\operatorname{dom} f(0) \cap$ GTE-dom $(\inf f, \overline{\mathbb{R}}(r))$.

(17) Let $f$ be a sequence of partial functions from $X$ into $\overline{\mathbb{R}}$ with the same dom, $F$ be a sequence of subsets of $S$, and $r$ be a real number. Suppose that for every natural number $n$ holds $F(n)=\operatorname{dom} f(0) \cap \mathrm{GT}$-dom $(f(n), \overline{\mathbb{R}}(r))$. Let $n$ be a natural number. Then (the superior set sequence of $F)(n)=$ $\operatorname{dom} f(0) \cap \mathrm{GT}$-dom( $($ the superior real sequence of $f)(n), \overline{\mathbb{R}}(r))$.

(18) Let $f$ be a sequence of partial functions from $X$ into $\overline{\mathbb{R}}$ with the same dom, $F$ be a sequence of subsets of $S$, and $r$ be a real number. Suppose that for every natural number $n$ holds $F(n)=\operatorname{dom} f(0) \cap$ GTE-dom $(f(n), \overline{\mathbb{R}}(r))$. Let $n$ be a natural number. Then (the inferior set sequence of $F)(n)=\operatorname{dom} f(0) \cap \mathrm{GTE}-\operatorname{dom}(($ the inferior real sequence of $f)(n), \overline{\mathbb{R}}(r))$.

(19) Let $f$ be a sequence of partial functions from $X$ into $\overline{\mathbb{R}}$ with the same dom and $E$ be an element of $S$. Suppose $\operatorname{dom} f(0)=E$ and for every 
natural number $n$ holds $f(n)$ is measurable on $E$. Let given $n$. Then (the superior real sequence of $f)(n)$ is measurable on $E$.

(20) Let $f$ be a sequence of partial functions from $X$ into $\overline{\mathbb{R}}$ with the same dom and $E$ be an element of $S$. Suppose $\operatorname{dom} f(0)=E$ and for every natural number $n$ holds $f(n)$ is measurable on $E$. Let $n$ be a natural number. Then (the inferior real sequence of $f)(n)$ is measurable on $E$.

(21) Let $f$ be a sequence of partial functions from $X$ into $\overline{\mathbb{R}}, F$ be a sequence of subsets of $S$, and $r$ be a real number. Suppose that for every natural number $n$ holds $F(n)=\operatorname{dom} f(0) \cap$ GTE-dom((the superior real sequence of $f)(n), \overline{\mathbb{R}}(r))$. Then $\bigcap F=\operatorname{dom} f(0) \cap$ GTE-dom $(\limsup f, \overline{\mathbb{R}}(r))$.

(22) Let $f$ be a sequence of partial functions from $X$ into $\overline{\mathbb{R}}, F$ be a sequence of subsets of $S$, and $r$ be a real number. Suppose that for every natural number $n$ holds $F(n)=\operatorname{dom} f(0) \cap$ GT-dom((the inferior real sequence of $f)(n), \overline{\mathbb{R}}(r))$. Then $\bigcup \operatorname{rng} F=\operatorname{dom} f(0) \cap \mathrm{GT}-\operatorname{dom}(\liminf f, \overline{\mathbb{R}}(r))$.

(23) Let $f$ be a sequence of partial functions from $X$ into $\overline{\mathbb{R}}$ with the same dom and $E$ be an element of $S$. Suppose $\operatorname{dom} f(0)=E$ and for every natural number $n$ holds $f(n)$ is measurable on $E$. Then $\lim \sup f$ is measurable on E.

(24) Let $f$ be a sequence of partial functions from $X$ into $\overline{\mathbb{R}}$ with the same dom and $E$ be an element of $S$. Suppose $\operatorname{dom} f(0)=E$ and for every natural number $n$ holds $f(n)$ is measurable on $E$. Then $\liminf f$ is measurable on E.

(25) Let $f$ be a sequence of partial functions from $X$ into $\overline{\mathbb{R}}$ with the same dom and $E$ be an element of $S$. Suppose that

(i) $\operatorname{dom} f(0)=E$,

(ii) for every natural number $n$ holds $f(n)$ is measurable on $E$, and

(iii) for every element $x$ of $X$ such that $x \in E$ holds $f \# x$ is convergent.

Then $\lim f$ is measurable on $E$.

(26) Let $f$ be a sequence of partial functions from $X$ into $\overline{\mathbb{R}}$ with the same dom, $g$ be a partial function from $X$ to $\overline{\mathbb{R}}$, and $E$ be an element of $S$. Suppose that

(i) $\operatorname{dom} f(0)=E$,

(ii) for every natural number $n$ holds $f(n)$ is measurable on $E$,

(iii) $\operatorname{dom} g=E$, and

(iv) for every element $x$ of $X$ such that $x \in E$ holds $f \# x$ is convergent and $g(x)=\lim (f \# x)$.

Then $g$ is measurable on $E$.

(27) Let $f$ be a sequence of partial functions from $X$ into $\overline{\mathbb{R}}$ and $g$ be a partial function from $X$ to $\overline{\mathbb{R}}$. Suppose that for every element $x$ of $X$ such that $x \in \operatorname{dom} g$ holds $f \# x$ is convergent to finite number and $g(x)=\lim (f \# x)$. Then $g$ is finite. 


\section{EgOROFF's ThEOREM}

The following three propositions are true:

(28) Let $M$ be a $\sigma$-measure on $S, f$ be a sequence of partial functions from $X$ into $\overline{\mathbb{R}}$ with the same dom, $g$ be a partial function from $X$ to $\overline{\mathbb{R}}$, and $E$ be an element of $S$. Suppose that

(i) $M(E)<+\infty$,

(ii) $\operatorname{dom} f(0)=E$,

(iii) for every natural number $n$ holds $f(n)$ is measurable on $E$ and $f(n)$ is finite,

(iv) $\operatorname{dom} g=E$, and

(v) for every element $x$ of $X$ such that $x \in E$ holds $f \# x$ is convergent to finite number and $g(x)=\lim (f \# x)$.

Let $r, e$ be real numbers. Suppose $0<r$ and $0<e$. Then there exists an element $H$ of $S$ and there exists a natural number $N$ such that

(vi) $H \subseteq E$,

(vii) $M(H)<r$, and

(viii) for every natural number $k$ such that $N<k$ and for every element $x$ of $X$ such that $x \in E \backslash H$ holds $|f(k)(x)-g(x)|<e$.

(29) Let $X, Y$ be non empty sets, $E$ be a set, and $F, G$ be functions from $X$ into $Y$. If for every element $x$ of $X$ holds $G(x)=E \backslash F(x)$, then $\bigcup \operatorname{rng} G=E \backslash \bigcap \operatorname{rng} F$.

(30) Let $M$ be a $\sigma$-measure on $S, f$ be a sequence of partial functions from $X$ into $\overline{\mathbb{R}}$ with the same dom, $g$ be a partial function from $X$ to $\overline{\mathbb{R}}$, and $E$ be an element of $S$. Suppose that

(i) $\operatorname{dom} f(0)=E$,

(ii) for every natural number $n$ holds $f(n)$ is measurable on $E$,

(iii) $M(E)<+\infty$,

(iv) for every natural number $n$ there exists an element $L$ of $S$ such that $L \subseteq E$ and $M(E \backslash L)=0$ and for every element $x$ of $X$ such that $x \in L$ holds $|f(n)(x)|<+\infty$, and

(v) there exists an element $G$ of $S$ such that $G \subseteq E$ and $M(E \backslash G)=0$ and for every element $x$ of $X$ such that $x \in E$ holds $f \# x$ is convergent to finite number and $\operatorname{dom} g=E$ and for every element $x$ of $X$ such that $x \in G$ holds $g(x)=\lim (f \# x)$.

Let $e$ be a real number. Suppose $0<e$. Then there exists an element $F$ of $S$ such that

(vi) $F \subseteq E$,

(vii) $\quad M(E \backslash F) \leq e$, and

(viii) for every real number $p$ such that $0<p$ there exists a natural number $N$ such that for every natural number $n$ such that $N<n$ and for every element $x$ of $X$ such that $x \in F$ holds $|f(n)(x)-g(x)|<p$. 


\section{REFERENCES}

[1] Grzegorz Bancerek. The fundamental properties of natural numbers. Formalized Mathematics, 1(1):41-46, 1990.

[2] Grzegorz Bancerek. König's theorem. Formalized Mathematics, 1(3):589-593, 1990.

3] Grzegorz Bancerek. The ordinal numbers. Formalized Mathematics, 1(1):91-96, 1990.

[4] Józef Białas. Series of positive real numbers. Measure theory. Formalized Mathematics, 2(1):173-183, 1991.

[5] Józef Białas. The $\sigma$-additive measure theory. Formalized Mathematics, 2(2):263-270, 1991

[6] Józef Białas. Some properties of the intervals. Formalized Mathematics, 5(1):21-26, 1996.

[7] Czesław Byliński. Functions from a set to a set. Formalized Mathematics, 1(1):153-164, 1990.

[8] Czesław Byliński. Partial functions. Formalized Mathematics, 1(2):357-367, 1990.

[9] Noboru Endou and Yasunari Shidama. Integral of measurable function. Formalized Mathematics, 14(2):53-70, 2006.

[10] Noboru Endou, Katsumi Wasaki, and Yasunari Shidama. Basic properties of extended real numbers. Formalized Mathematics, 9(3):491-494, 2001.

[11] Noboru Endou, Katsumi Wasaki, and Yasunari Shidama. Definitions and basic properties of measurable functions. Formalized Mathematics, 9(3):495-500, 2001.

[12] Adam Grabowski. On the Kuratowski limit operators. Formalized Mathematics, 11(4):399-409, 2003.

[13] P. R. Halmos. Measure Theory. Springer-Verlag, 1987.

[14] Jarosław Kotowicz and Yuji Sakai. Properties of partial functions from a domain to the set of real numbers. Formalized Mathematics, 3(2):279-288, 1992.

[15] Andrzej Nẹdzusiak. $\sigma$-fields and probability. Formalized Mathematics, 1(2):401-407, 1990.

[16] Beata Padlewska. Families of sets. Formalized Mathematics, 1(1):147-152, 1990.

[17] Beata Perkowska. Functional sequence from a domain to a domain. Formalized Mathematics, 3(1):17-21, 1992.

[18] Zinaida Trybulec. Properties of subsets. Formalized Mathematics, 1(1):67-71, 1990.

[19] Edmund Woronowicz. Relations and their basic properties. Formalized Mathematics, 1(1):73-83, 1990.

[20] Edmund Woronowicz. Relations defined on sets. Formalized Mathematics, 1(1):181-186, 1990.

[21] Hiroshi Yamazaki, Noboru Endou, Yasunari Shidama, and Hiroyuki Okazaki. Inferior limit, superior limit and convergence of sequences of extended real numbers. Formalized Mathematics, 15(4):231-236, 2007.

[22] Bo Zhang, Hiroshi Yamazaki, and Yatsuka Nakamura. Limit of sequence of subsets. Formalized Mathematics, 13(2):347-352, 2005. 